\title{
Gestión y efectividad en educación: evidencias comparativas entre establecimientos municipales y particulares subvencionados*
}

\author{
Management and efficiency in education: comparative evidences \\ between municipal and private subsidized establishments \\ Gestão e efetividade em educação: evidências comparativas entre \\ estabelecimentos municipais e particulares subvencionados
}

\author{
Patricia Castilloํ, Alejandra González², Ismael Puga \\ ${ }^{1}$ Doctora $($ C en Psicología, Universidad Paris VIII, Magíster en Psicoanálisis, Universidad de Buenos Aires, \\ Psicóloga Pontificia Universidad Católica de Chile. Académica Departamento de Trabajo Social, \\ Universidad Alberto Hurtado. Fono: 02-8897470. E-mail: pcastilg@uc.cl \\ ${ }^{2}$ Magíster en Trabajo Social, Pontificia Universidad Católica de Chile, Trabajadora Social, \\ Pontificia Universidad Católica de Chile. Académica Universidad Alberto Hurtado. \\ Fono: 02-8897470. E-mail: algonzal@uahurtado.cl \\ ${ }^{3}$ Doctor (C) en Sociología, Universidad Humboldt, Alemania, Magíster en Ciencias Sociales FLACSO \\ México, Sociólogo, Universidad de Chile, Colaborador Universidad Alberto Hurtado. \\ Fono: 02-8897470. E-mail: ismael.puga@gmail.com
}

\begin{abstract}
RESUMEN
El siguiente artículo contrasta mediante una estrategia cuantitativa la realidad de tres hipótesis que hoy postulan la necesidad de acrecentar la participación de los establecimientos de administración privada en Chile. Las principales conclusiones son: la diversidad que aportan las escuelas particulares subvencionadas es la de segmentar según estrato socioeconómico sus objetivos, misiones y visiones, favoreciendo en los sectores medios-bajos y bajos la entrega de hábitos y disciplina por sobre la excelencia académica; los establecimientos municipales en los sectores medios-bajos y bajos entregan una educación más efectiva en términos de resultados escolares que los particulares subvencionados. En las variables de gestión no se encontraron diferencias significativas según dependencia y en muchas de las variables las pequeñas diferencias favorecían la gestión de tipo municipal.
\end{abstract}

Palabras clave: desempeño escolar, gestión escolar, educación municipal y particular subvencionada, efectividad escolar, eficiencia escolar.

\begin{abstract}
By means of a quantitative strategy this study contrasts the reality of three hypotheses that nowadays claim the need to add the participation of the privately administered schools in Chile. The main conclusions are: the diversity contributed by the private subsidized schools is only to segment their objectives, missions and visions according to the socio-economic status. Furthermore, they favor the acquisition of habits and discipline instead of the academic excellence in the middle-lower and lower sectors. Compared to the private subsidized schools,
\end{abstract}

* Este artículo tiene como origen el estudio “Municipalización, Organización Escolar y Autonomía. Indicadores para una Gestión Escolar de Calidad” financiado por el Fondo de Investigación y Desarrollo de la Educación dependiente del Ministerio de Educación y el Departamento de Ingeniería Industrial de la Universidad de Chile, realizado entre los años 2007-2008, y cuyo objetivo fue indagar los elementos relacionados con una gestión escolar asociada a buenos resultados escolares. Ese estudio tuvo como muestra 235 establecimientos de la Región Metropolitana pertenecientes a administración municipal, administración particular subvencionada; estratos socioeconómicos bajo, medio bajo y medio alto y de buen y mal rendimiento. 
the municipal establishments in the middle-lower and lower sectors offer a more effective education with regard to the school results There are not significant differences between both school systems and in many variables the small differences favored the municipal schools.

Key words: school performance, school management, municipal and particular subsidized education, school efficiency.

\section{RESUMO}

O seguinte artigo contrasta mediante uma estratégia quantitativa a realidade de três hipóteses que hoje postulam a necessidade de acrescentar a participação dos estabelecimentos de administração privada no Chile. As principais conclusões são: a diversidade que oferecem as escolas particulares subvencionadas é a de segmentar os seus objetivos, missões e visões, dependendo do estrato socioeconômico, favorecendo a antrega de hábitos e disciplina por sobre a excelência acadêmica nos setores médio-baixos e baixos. Os estabelecimentos municipais nos setores médio-baixos e baixos entregam uma educação mais eficiente em quanto aos resultados escolares que os particulares subvencionados. Nas variáveis da gestão não encontram-se diferenças significativas em relaçao à dependência e em muitas das variáveis as pequenas diferenças favoreceram a gestão de tipo municipal.

Palavras-chave: desempenho escolar, gestão escolar, educação municipal e particular subvencionada, efetividade escolar, eficiencia escolar.

\section{INTRODUCCION}

Durante el año 2007-2008 fue realizado el estudio "Municipalización, Organización Escolar y Autonomía. Indicadores para una Gestión Escolar de Calidad" que abarcó 235 escuelas de la Región Metropolitana. El objetivo de dicha investigación era encontrar los elementos de la gestión escolar (en adelante GE) que tenían la más alta relación con desempeño escolar.

En esa investigación se recogieron exhaustivamente datos relacionados con la gestión escolar en establecimientos de distinta dependencia y distinto nivel socioeconómico (en adelante NSE). Al realizar dicho estudio se encontró escasa evidencia sobre gestión escolar que contara con un respaldo estadístico que permitiera generalizar resultados de una manera segura y fue por ello que se decidió hacer una segunda lectura de los resultados para determinar la veracidad de algunas afirmaciones sobre la diferencia de gestión y resultados entre establecimientos de dependencia municipal (en adelante MUN) o particulares subvencionados (en adelante PS). (Eguiguren 2009).

$\mathrm{Al}$ respecto, fue posible contrastar tres afirmaciones que aparecen con frecuencia en el discurso de los distintos actores que participan en la política educativa del país:

1. Las escuelas PS aportan una mayor diversidad a la educación en general.

2. Las escuelas PS obtienen mejores resultados que las MUN, dado el mismo NSE.

3. Las escuelas PS realizan una mejor gestión que las MUN, dado el mismo NSE.

Al revisar la bibliografía existente sobre gestión escolar es posible encontrar que cada estudio hace explícita la dificultad técnica de realizar una comparación justa entre establecimientos de dependencia MUN y PS, debido a la dificultad de controlar de manera fina las variables asociadas al distinto NSE; se agrega a ello la dificultad de neutralizar aquellos aspectos que están relacionados con los mecanismos de selección y exclusión de estudiantes que los establecimientos PS realizan durante el año, con lo cual la comparación adquiere ribetes muy complejos, pues claramente no estaríamos ante instituciones sometidas a la misma normativa. 
Un ejemplo de esto es lo planteado por Mizala y Romaguera (1998) al citar un estudio de Rounds Parry (1996) quien al entrevistar a una muestra aleatoria de establecimientos educacionales de Santiago encontró que los colegios particulares subvencionados recurrían en mayor medida a exámenes, exigencias de notas mínimas y entrevistas a los padres en su proceso de selección de estudiantes. Esto significa que esos establecimientos aceptan estudiantes que rinden mejor (que tienen más habilidades), característica que no es controlada por las variables disponibles (Mizala, Romaguera, Farren 1998).

Sin embargo, aun con estas dificultades, es posible percibir en la mayor parte de los estudios $^{1}$ sobre efectividad entre los distintos establecimientos que los colegios MUN y PS en los estratos medios bajos y bajos se diferencian escasamente y cuando se diferencian son los establecimientos municipales los que obtendrían la ventaja, no solo en rendimiento, sino también en gestión de recursos, adquisición de capacidades técnicas y compensación de dificultades socioeconómicas y familiares de los estudiantes.

Este artículo intentará aportar a esa discusión, analizando evidencia empírica que permita avanzar en el conocimiento de estas variables.

\section{LO PUBLICO Y LO PRIVADO EN LA EDUCACION EN CHILE}

A inicios de los 80, bajo la dictadura militar, las escuelas públicas, dependientes hasta entonces del Ministerio de Educación, son transferidas a los gobiernos locales dando origen al sector municipalizado de educación. En ese mismo lapso se autorizó el financiamiento de parte del Estado a colegios con la condición de que no cobraran pagos de matrícula a los estudiantes, denominándose a esas escuelas particulares subvencionadas.

Los administradores de las escuelas reciben de parte del Estado una subvención o pago por estudiante. La asignación de los recursos entre las escuelas se subordina a las decisiones que las familias realizan respecto del lugar de estudios de sus hijos, en un contexto donde los oferentes educacionales compiten para atraer y retener a los estudiantes. El sistema de la subvención se asemeja a un esquema de vouchers ${ }^{2}$, aun cuando sea el Estado el que transfiere los recursos financieros a los establecimientos en función de la matrícula que asiste a clases.

1 Ver los estudios de: González, P.; Mizala, A. y Romaguera, P., 2001; Mizala, A. y Romaguera, P., 2000; Mizala, A. y Romaguera, P., 2002; Mizala, A. y Romaguera, P., 2001; Brunner, J. J. y Elacqua, G., 2003; OPECH 2006; Redondo, J.M.; Descouvieres, C. 2000; Aedo, C. y Sapelli, C., 2001; Sapelli, C. \& Torche, A. 2002; Tokman, A., 2002; Gallego, F.A., 2002; Carnoy, M., 2005; Elacqua, G., 2004; García-Huidobro, J. E.; Bellei, C., 2006; López, N., 2005; Chávez, 2006; Redondo, 2005; Larrañaga, O., 2004.

2 Según Oscar Bonilla en "El Voucher Educacional” (1995). El esquema del voucher fue propuesto por primera vez para la educación en 1955 por el profesor Milton Friedman de la Universidad de Chicago. Se basa en la distribución de vouchers educacionales a todos los padres de familia. Estos vouchers son cupones canjeables por educación en las diversas escuelas, públicas o privadas, aprobadas por el Ministerio de Educación. El valor del voucher podría ser calculado en función del costo promedio de educación para un determinado nivel académico. Dicho valor será pagado por el Estado a las escuelas, previa entrega de los vouchers aceptados por éstas como pago por sus servicios. Si un padre de familia deseara brindar a su hijo una educación de un costo superior, el valor del voucher podría ser complementado con un pago adicional.

Por las características de funcionamiento del voucher, éste debería ser un beneficio intransferible y canjeable solamente por educación. Estaría relacionado con un niño específico y sería aceptado únicamente en las escuelas participantes del proyecto. Estas escuelas serían supervisadas cada cierto tiempo para garantizar que no estén por debajo de un cierto estándar educacional y que sean competentes para proporcionar el servicio. 
Larrañaga (1994) distingue tres períodos en los últimos 30 años de este sistema:

1. El primero, de gran expansión de parte del sector privado: cerca de un $15 \%$ del total de los estudiantes del país se trasladan desde las escuelas públicas a las privadas subvencionadas en el período 1981-1986, causando un aumento de la participación en la matrícula del sector de $15,1 \%$ a $30,8 \%$. El número de establecimientos del sector privado subvencionado crece en un $62 \%$ entre 1980 y 1985. Por su parte, las escuelas públicas experimentan una caída en su participación de 78,0\% a 63,1\% en el período 1981-1986, aun cuando el número de sus establecimientos se mantiene relativamente constante.

2. Un período de relativo estancamiento, desde el año 1986 a 1994: se caracteriza por una fuerte disminución de la inversión pública en educación (baja la subvención). El retorno a las instituciones democráticas empuja el diseño de políticas de educación que tienden a recuperar el rol del Estado como rector del sistema de Educación y no el mercado. Se sancionan distintas leyes que hacen menos lucrativa la inversión privada en educación.

3. 1994 hasta la fecha. Se reactiva la competencia producto de la entrada en vigencia del financiamiento compartido, que permite a los privados agregar valor a la subvención cobrándoles una cuota compensatoria a los padres de cada estudiante. El número de establecimientos particulares subvencionados alcanza hoy el $42 \%$ del total de la matrícula. A su vez, en el año 1995 se relajan algunas de las restricciones a la movilidad laboral, permitiéndose a los municipios modificar la dotación de profesores frente a cambios en la matrícula de estudiantes, sujeto a la condición que sean profesores que tengan contratos de carácter transitorio. Ese mismo año se introduce un esquema de incentivos (en adelante SNED) que favorece a los profesores de los colegios con mejores resultados medidos a través de tres indicadores que combinan rendimiento, valor agregado e igualdad de oportunidades.

En esta misma dirección el año 2008 se inicia la implementación de la Ley de Subvención Escolar Preferencial (en adelante SEP) que crea una subvención preferencial para los estudiantes más pobres y un sistema de control y sanciones para las escuelas, basado en los resultados de aprendizaje de los estudiantes. Tal como lo indica su nombre esta subvención del Estado es una subvención a la demanda, que determina en forma individual los casos que por situación socioeconómica y familiar sufren los mayores efectos de la inequidad, asignándoles un diferencial de recursos para que los establecimientos inviertan ese dinero en obtener las condiciones necesarias para compensar las dificultades de origen que traen estos estudiantes.

\section{LOS ESTUDIOS COMPARATIVOS SOBRE EFECTIVIDAD Y GESTION ESCOLAR}

Es posible afirmar que los estudios existentes se han concentrado en evaluar el rendimiento de las escuelas, o bien el rendimiento de la educación en general a partir de la existencia de este sistema y la influencia de los distintos tipos de incentivos del 
Estado para el mejoramiento de la educación en cada uno de estos subsistemas o en su conjunto. Los resultados son diversos y difícilmente definitivos:

Por un lado, la imposibilidad de medir mecanismos conocidos, pero pocas veces admitidos como la selección de estudiantes, la expulsión según rendimiento o disciplina u otras medidas que desregulan finalmente aquello que se indica como libre competencia entre establecimientos municipales y subvencionados, hace complejo establecer juicios sobre el desempeño y la efectividad de este sistema. Entonces, si bien algunos estudios indican una diferencia en el rendimiento de los establecimientos que favorece a la administración particular subvencionada, todos los estudios mencionan el efecto distorsionador de los mecanismos mencionados.

Por otra parte, la relación entre las condiciones socioeconómicas de la familia -con todos los efectos que esto puede tener sobre las condiciones de aprendizaje- y la dependencia de la escuela implica que la diferencia en el desempeño de las escuelas de distinto tipo no puede explicarse únicamente por variables internas: las escuelas municipales son comparadas permanentemente con establecimientos donde los estudiantes tienen un mayor apoyo de parte de los padres, menores dificultades de aprendizaje, un desarrollo madurativo acorde con el curso en el que se encuentran, los padres poseen un nivel educacional superior, etc.

Aún así, los estudios muestran diferencias de rendimiento mínimas entre una modalidad y otra.

Según la OPECH (Descouvieres, Redondo, Rojas, 2004), la única región en donde es posible pesquisar diferencias estadísticamente significativas entre la educación municipal y la educación particular subvencionada es la Región Metropolitana y establecen que la mayoría de esa diferencia estaría relacionada con la política de selección de alumnos que se aplica con mayor frecuencia en la Región Metropolitana.

En el mismo estudio de la OPECH, referido a la eficiencia de los centros de enseñanza media de Chile en la segunda parte de la década de los 90, se indica claramente que "no es cierto que la distancia de calidad (entendida solamente como resultados escolares medidos por el SIMCE) entre los centros, cuando se neutralizan las condiciones externas de input, sea tan extremadamente favorable al sector privado" (Descouvieres, Redondo, Rojas, 2004: 56). Además indican que la evolución longitudinal de los datos arrojaría evidencias de que los centros que han mejorado en este período han sido los subvencionados con fondos públicos, y especialmente los municipales. Es importante este dato, pues permite observar cómo la gestión pública va mejorando e impactando en los resultados.

Respecto a los aspectos determinantes en la explicación de los resultados escolares, "está determinada en la enseñanza media chilena en un 70\% por los factores externos a las escuelas e internos a las familias. Al mismo tiempo las escuelas hacen la diferencia en ese otro $30 \%$. De forma que los centros hacen la diferencia independientemente de su dependencia jurídica. Hay centros eficientes entre los particulares y entre los municipales; también ineficientes en ambos grupos" (Descouvieres, Redondo, Rojas, 2004: 56).

Otro dato interesante de este estudio es que en los establecimientos de enseñanza media particulares subvencionados se produce una mayor correlación entre el nivel educacional de los padres y los resultados escolares que en los establecimientos municipales; esto implicaría que los centros educativos municipales logran una mayor compensación de los factores socioeconómicos y familiares, en igualdad de inversión con fondos públicos. 
Mizala y Romanguera (1998) realizan a su vez un estudio en el que comparan la eficiencia técnica ${ }^{3}$ de los distintos establecimientos educacionales, para lo cual evaluaron dos metodologías ${ }^{4}$ : en la primera no encontraron diferencias entre los establecimientos particulares subvencionados y los de administración municipal, en el segundo método utilizado habría una leve diferencia que favorece a los particulares subvencionados. Sin embargo, en las conclusiones aparecen todas las dificultades técnicas (control de factores socioeconómicos, la selección de estudiantes y la expulsión de los estudiantes de bajo rendimiento, entrevista a los padres, etc.) que significa comparar establecimientos escolares en los cuales no rigen las mismas normas.

Es importante resaltar en este punto el tema del estatuto docente y el aporte o la diferencia que éste tiene en la eficiencia escolar; en ello no hay hasta ahora ningún estudio que indique diferencias sustanciales en el desempeño de profesores en los distintos tipos de establecimientos. De hecho, un estudio realizado por Mizala y Romaguera (2000) que indaga en la experiencia docente y los incentivos concluye: "A pesar de la distinta institucionalidad a la que están sujetos los docentes, no se observan diferencias significativas entre los distintos tipos de establecimientos educacionales con respecto al desempeño de los maestros".

Respecto de los resultados del sistema educacional en Chile, el informe OCDE indica: “...los estudiantes de colegios particulares de $4^{\circ}$ y $8^{\circ}$ básico no hicieron mayores avances que los estudiantes de establecimientos públicos (durante la década de los '90)” (pp. 181-183). Es más, “..es sorprendente que la evidencia empírica de los años '90 revele que los estudiantes de un estatus socioeconómico similar obtienen un puntaje algo menor en los colegios particulares subvencionados laicos (cerca de dos tercios de todos los establecimientos particulares subvencionados) que en los establecimientos municipales" (p. 185).

Esto pone en cuestión todo el sistema de incentivos a la competencia que han caracterizado las políticas públicas en educación, tal como lo establecen en otro artículo García-Huidobro, J. E.; Bellei, C. (2006) que se pronuncian sobre la subvención diferenciada: "tampoco está clara la efectividad educativa de la teoría del premio/castigo. La experiencia chilena de libre competencia ha sido paradigmática a este respecto: aunque las escuelas compiten activamente en el mercado del prestigio escolar, los resultados de aprendizaje de los estudiantes no mejoran. Adicionalmente, las escuelas que pierden inician penosos procesos de deterioro institucional, social y académico. En otras palabras, el mercado puede crear un sistema escolar altamente dinámico, pero no por ello de mejor calidad".

Elacqua, G. (2004), cuando se refiere a los estudios que avalan la teoría de la competencia como herramienta para mejorar la calidad pero sobre todo para resolver el problema de la equidad en la educación, indica que "los estudios en Chile muestran que se ha producido un pequeño impacto positivo de la competencia de escuelas privadas en el logro académico de las escuelas públicas. Sin embargo, la evidencia no es concluyente.

3 Eficiencia técnica se entiende como la Capacidad de los Establecimientos de Generar el Máximo Producto (Logro Educativo) Dada su Combinación de Insumos.

4 La estimación de una frontera estocástica de producción de donde se derivan coeficientes de eficiencia, y el Data Envelopment Analysis (DEA) que es una forma no paramétrica de identificar la frontera de producción eficiente de una unidad productiva. 
Por ejemplo, McEwan y Carnoy (2000) demuestran que la competencia sólo ha producido modestos logros en las escuelas públicas en Santiago y que, por el contrario, ha producido efectos levemente negativos fuera de la capital. Hsieh y Urquiola (2003) llegan a conclusiones similares; sin embargo, Gallego (2002) y Auguste y Valenzuela (2003) sostienen que sus resultados son revertidos si se usan variables instrumentales para dar cuenta del porcentaje de estudiantes privados en la municipalidad". Es decir, sobre este aspecto no hay ningún consenso establecido.

En el mismo estudio Elacqua establece: "Hsieh y Urquiola (2003) muestran que la brecha entre el sector subsidiado y el privado no subsidiado ha aumentado entre $1982 \mathrm{y}$ 1996. Al agregar el año 2002 se puede observar que la brecha entre escuelas subsidiadas y no subsidiadas aumenta aún más. Esto confirma que el sistema de libre elección no tiene efectos positivos en los logros académicos de las escuelas subsidiadas. Es decir, la competencia no ha mejorado los resultados del sector subsidiado" (Elacqua, G. 2004: 9).

En términos generales, la experiencia chilena parece demostrar que las políticas de libre elección de escuelas favorecen a familias de ingresos medios y alto y que en los sectores más desfavorecidos este sistema solo ha ido en desmedro de la calidad de la educación de los establecimientos públicos, pues son estos quienes deben entregar educación a todos los estudiantes que el sistema particular subvencionado no acepta o expulsa, provocando que los grupos de estudiantes pertenecientes al sistema público sean homogéneamente los más excluidos, los con más problemas, los con menos soportes y además, producto de la segmentación, los que quedan imposibilitados de ser contenidos por los pares que se encuentran en mejores condiciones.

Al respecto, Sapelli, C. \& Torche, A. (2002) plantean que los resultados de las pruebas SIMCE de castellano y matemáticas son mejores en los colegios subvencionados y particulares por sobre los de administración municipal; sin embargo, en su análisis se refieren transversalmente a los establecimientos, sin distinguir por nivel socioeconómico, lo cual permite cuestionar su conclusión. Es interesante, pues en el mismo estudio se afirma que "el $79 \%$ de los menores del primer quintil que viven en hogares en que el jefe de hogar no tiene educación están en establecimientos municipalizados, en tanto que sólo el 59\% de los estudiantes del mismo quintil pero cuyos padres tienen educación media están en escuelas municipalizadas. En general, puede observarse que para cada quintil la adscripción a las escuelas municipalizadas se reduce conforme aumenta el nivel de escolaridad del padre. Por otra parte, cualquiera sea el nivel educacional del padre, la adscripción en términos porcentuales a escuelas municipalizadas se reduce conforme aumenta el nivel de ingreso del hogar" (Sapelli, C. \& Torche, A. 2002: 181). Es decir, parece evidente no solo la composición de los establecimientos educacionales de los quintiles más pobres, sino también los factores familiares que están presentes en los establecimientos municipalizados pertenecientes a este quintil.

Por último, el estudio de Tokman (2002) "Is private education better? evidence from Chile" sugiere en sus resultados que los colegios públicos no serían ni uniformemente peor ni mejor que los privados. Más aún, los colegios públicos serían relativamente más efectivos para estudiantes de familias de estratos socioeconómicos más bajos.

Es decir, la educación impartida entre escuelas subvencionadas y escuelas municipales no ha sido exhaustivamente comparada; las dificultades técnicas que devienen de las diferencias económicas y sociales, así como de los distintos mecanismos de selección y 
exclusión que utilizan los establecimientos subvencionados dificultan la tarea de evaluar de manera justa esta relación.

\section{LA GESTION ESCOLAR}

La gestión escolar involucra la distribución de los roles y las relaciones que se generan en el conjunto de los actores educacionales. Se entenderá gestión escolar como "un conjunto de acciones articuladas entre sí, que posibilitan la consecución de la intencionalidad de la institución (...) conjunto de articulaciones que se dan al interior de la escuela entre el equipo directivo, los docentes, los estudiantes y los padres y apoderados" (Zúñiga, 2005).

Considerando que la gestión escolar constituye una variable que se entiende como clave para la efectividad, el presente artículo se centra en la dimensión de organización escolar y los rendimientos asociados a los distintos tipos de establecimientos.

\section{ORGANIZACION ESCOLAR}

La organización escolar no es sólo una estructura formal, "sino también el cómo se utiliza realmente ésta, qué relaciones se potencian y se desarrollan entre sus miembros; cómo se abordan y se llevan a cabo los procesos organizativos, qué valores se cultivan y expresan en la práctica cotidiana del centro, qué relaciones, cómo y por qué se mantienen con la comunidad y el entorno, y cómo todo ello contribuye o dificulta el desarrollo de procesos educativos ricos y valiosos para los estudiantes" (González 2003: 32).

Por otra parte, la reciente literatura sobre escuelas efectivas destaca la importancia de una buena gestión para el éxito de los establecimientos (Alvariño et al., 2000). En ésta se constata la existencia de una serie de factores relevantes de considerar en los análisis de las organizaciones escolares de altos desempeños, ya señalados, que en síntesis serían:

- Una visión institucional clara y compartida por todos.

- Una cultura escolar basada en el compromiso hacia la escuela y hacia el estudiante, en la responsabilidad de los profesionales, en la colaboración y comunicación entre los pares, en el respeto y en el apoyo hacia la innovación.

- Una organización que cuenta con los recursos mínimos para el fomento de la educación y que se preocupa del proceso de enseñanza y aprendizaje mediante el perfeccionamiento de sus profesores como a través del intercambio de experiencias.

- Una organización que dedica tiempo y procura los espacios para el trabajo profesional en equipo, y en donde las decisiones pedagógicas importantes se discuten entre todos dejando al mismo tiempo libertad para que el profesor pueda innovar en sus clases.

- Una organización que despliega diversas estrategias de reflexión y acción sobre su quehacer cotidiano.

- El liderazgo académico de los distintos actores, especialmente del director del establecimiento. 
- Las políticas organizacionales y el desarrollo de estrategias que tengan relación con los fines del centro educativo, en el marco de la disponibilidad y utilización de espacios y recursos.

- El espacio donde conviven prácticas y creencias de los actores que forman parte de la organización dentro de una estructura formal de trabajo.

- La distribución jerárquica de funciones y roles de distintos actores.

\section{ASPECTOS COMPARADOS ENTRE ESTABLECIMIENTOS MUNICIPALIZADOS Y PARTICULARES SUBVENCIONADOS}

En este estudio se compararon datos buscando responder a las afirmaciones anteriormente mencionadas, por tanto se buscó:

- Establecer la veracidad respecto al aporte de diversidad de proyectos educativos a partir de la existencia de establecimientos particulares subvencionados. Para ello se evaluaron tres aspectos: la misión, pues ella entrega una idea importante respecto a los objetivos formales de los establecimientos; la operacionalización de esta misión, pues es una forma de saber algo más concreto en relación a lo planteado discursivamente en torno a los objetivos, y el estilo organizacional, que está en sincronía con el proyecto general que se plantean las instituciones. Todo esto debiera iluminar aquellos aspectos relacionados con el proyecto educativo y desde ahí poder evaluar la heterogeneidad de los mismos.

- Establecer la veracidad respecto al rendimiento académico obtenido por los establecimientos según dependencia despejando la variable asociada al nivel socioeconómico al que pertenece territorialmente cada institución. La variable dependiente, efectividad escolar, se operacionalizó como un puntaje asignado de rendimiento que se construyó considerando: 1) Puntaje obtenido en el último SIMCE para $8^{\circ}$ básico (2004); 2) Rezago de estudiantes dentro de cada escuela, y 3) Reprobación de estudiantes. Estos dos últimos factores se incluyen como expresiones de desempeño negativo, representando la incapacidad de proveer de las habilidades requeridas a parte de los alumnos. Cuando resultó necesario para el análisis, este indicador de intervalo fue codificado en categorías utilizando quintiles de desempeño. La determinación del nivel socioeconómico se realizó según la categorización SNED.

- Establecer la veracidad respecto a las diferencias en materia de gestión escolar según dependencia. Los elementos que caracterizan una buena gestión son múltiples y muchos se encuentran directamente relacionados a la disponibilidad de recursos en un establecimiento. Para comparar la capacidad de gestión de las escuelas se utilizaron algunos elementos que identifican con claridad buenas prácticas de gestión o resultados beneficiosos directamente asociados a una gestión eficiente. Estos incluyen: a) la evaluación sistemática de la actividad docente; b) el uso de evaluaciones técnico-pedagógicas de los establecimientos; y c) la obtención de recursos monetarios adicionales a las subvenciones a través de gestiones especiales; d) recursos para el fomento de la educación; e) instancias de perfeccionamiento para profesores; f) un fuerte liderazgo académico del director del establecimiento y g) los mecanismos de control e incentivos. 


\section{RESULTADOS}

\section{AFIRMACION 1:}

Las escuelas particulares subvencionadas aportan una mayor diversidad a la educación en general

Para comprobar esta posible diferencia se compararon las definiciones de las escuelas en términos de: a) la Misión; b) la Operacionalización de la Misión, y c) el Estilo Organizacional.

En términos de la definición de la Misión, existen diferencias claras entre MUN y PS. Las escuelas municipales tienden a enfatizar la formación ciudadana mientras que las escuelas PS se distribuyen con mayor variación entre esta opción y la de privilegiar una formación "valórica" o la formación de hábitos. En este sentido podría pensarse que existe mayor diversidad de proyectos educativos.

Sin embargo, si integramos la variable socioeconómica veremos que esta diferencia en la diversidad de las "misiones" solo podría apreciarse (y de manera no tan significativa) en el sector de NSE Bajo y NSE Medio-Bajo, donde la administración particular muestra una especial despreocupación por la excelencia académica. En cambio, las diferencias son menores en el NSE Medio, donde los colegios particular subvencionados comparten con los municipalizados la preocupación por la excelencia académica. Los colegios municipales tienden a ser más homogéneos en cuanto a Misión, concentrándose en formación ciudadana y la educación de excelencia. Es decir, finalmente la característica de las escuelas PS no parece ser una oferta de mayor diversidad, sino una oferta segmentada de acuerdo al nivel socioeconómico de los establecimientos.

\section{Cuadro 1}

Misión de las escuelas de distintas dependencias de NSE "Bajo" y "Medio Bajo".

(Según el SNED)

\begin{tabular}{|l|c|c|c|}
\hline \multicolumn{4}{|c|}{$\begin{array}{c}\text { Definición de la Misión según Dependencia: Frecuencias Relativas (\%). } \\
\text { Escuelas de NSE Bajo y Medio-Bajo }\end{array}$} \\
\hline \multicolumn{1}{|c|}{$\begin{array}{c}\text { Definición de la Misión } \\
\text { (Mención Principal) }\end{array}$} & $\begin{array}{c}\text { Escuelas } \\
\text { MUN }\end{array}$ & $\begin{array}{c}\text { Escuelas } \\
\text { PS }\end{array}$ & Total \\
\hline Entregar una educación de excelencia & 27,3 & 17,8 & 25,3 \\
\hline $\begin{array}{l}\text { Formar ciudadanos integrados, responsables } \\
\text { y participativos }\end{array}$ & 45,6 & 34,0 & 43,1 \\
\hline Entregar a sus estudiantes valores sólidos & 14,7 & 17,2 & 15,2 \\
\hline $\begin{array}{l}\text { Enseñar responsabilidad, autonomía y hábitos } \\
\text { de trabajo y estudio }\end{array}$ & 12,4 & 31,0 & 16,4 \\
\hline
\end{tabular}




\section{Cuadro 2}

Misión de las escuelas de distintas dependencias de NSE "Medio".

(Según el SNED)

\begin{tabular}{|l|c|c|c|}
\hline \multicolumn{4}{|c|}{$\begin{array}{c}\text { Definición de la Misión según Dependencia: Frecuencias Relativas (\%). } \\
\text { Escuelas de NSE Medio }\end{array}$} \\
\hline \multicolumn{1}{|c|}{$\begin{array}{c}\text { Definición de la Misión } \\
\text { (Mención Principal) }\end{array}$} & $\begin{array}{c}\text { Escuelas } \\
\text { MUN }\end{array}$ & $\begin{array}{c}\text { Ecuelas } \\
\text { PS }\end{array}$ & Total \\
\hline Entregar una educación de excelencia & 38,9 & 37,7 & 38,0 \\
\hline $\begin{array}{l}\text { Formar ciudadanos integrados, responsables y } \\
\text { participativos }\end{array}$ & 42,3 & 26,4 & 31,0 \\
\hline Entregar a sus alumnos valores sólidos & 6,6 & 26,1 & 20,5 \\
\hline $\begin{array}{l}\text { Enseñar responsabilidad, autonomía y hábitos de } \\
\text { trabajo y estudio }\end{array}$ & 10,0 & 9,8 & 9,9 \\
\hline Ninguna de las anteriores & 2,2 & 0,0 & 0,6 \\
\hline
\end{tabular}

Ahora bien, como se observa en la tabla siguiente, al preguntarse en qué se traduce concretamente la misión en los distintos establecimientos, la diferencia entre MUN y PS, aun sin introducir la variable socioeconómica, desaparece por completo.

\begin{tabular}{|l|r|r|r|}
\hline \multicolumn{4}{|c|}{$\begin{array}{c}\text { Operacionalización de la Misión según Dependencia: } \\
\text { Frecuencias Relativas (\%) }\end{array}$} \\
\hline $\begin{array}{c}\text { Operacionalización de la Misión } \\
\text { (Mención Principal) }\end{array}$ & $\begin{array}{c}\text { Escuelas } \\
\text { MUN }\end{array}$ & $\begin{array}{c}\text { Escuelas } \\
\text { PS }\end{array}$ & Total \\
\hline El tipo de actividades extraprogramáticas & 7,8 & 6,5 & 7,1 \\
\hline El trato (amistoso, respetuoso, cercano) con los estudiantes & 49,5 & 49,3 & 49,4 \\
\hline En la disciplina o en la autodisciplina & 5,9 & 10,2 & 8,3 \\
\hline En los niveles de exigencia académica & 25,6 & 27,9 & 26,9 \\
\hline La forma de resolución de conflictos & 11,2 & 6,1 & 8,4 \\
\hline
\end{tabular}

Es decir, a nivel de misión y de operacionalización de la misión, solo puede encontrarse una suerte de diversidad que de existir realmente iría en desmedro de los sectores más pobres de la sociedad, en los cuales la administración particular subvencionada no se impone otros desafíos que los de enseñar hábitos o aumentar la disciplina.

En este mismo ámbito existe la teoría de que los establecimientos según dependencia tendrían estilos organizacionales ${ }^{5}$ distintos; sin embargo, la diferencia encontrada indicaría

5 El estudio realizado recoge diversas variables sobre el estilo organizacional a través de una escala de acuerdo/desacuerdo respecto de diversas prácticas. Los directivos fueron consultados sobre su acuerdo con las siguientes afirmaciones: 1 . Los profesores confían en los directivos en general y en el director en particular; 2. Cuando criticas constructivamente el trabajo de alguien aquí, en general tienes buena recepción; 3 . Aunque sienta que un profesor está cometiendo un error, prefiero no entrometerme en su trabajo; 4. Los profesores 
que efectivamente existe más variedad al interior de las escuelas PS pero únicamente en el sentido de que existen más escuelas PS donde prima un estilo de gestión autoritario y menor involucramiento de los profesores (elementos generalmente minoritarios), lo cual a su vez estaría segmentado según nivel socioeconómico, concentrándose en los sectores bajos y medio-bajo los estilos organizacionales de mayor autoritarismo, mientras en los sectores medios nuevamente desaparecen las diferencias según dependencia.

\begin{tabular}{|l|c|c|c|c|c|c|c|}
\hline \multicolumn{8}{|c|}{$\begin{array}{c}\text { Estilo Organizacional según Dependencia: } \\
\text { Frecuencias Relativas de las Respuestas del Directivo (\%) }\end{array}$} \\
\hline \multirow{3}{*}{ NSE } & \multirow{2}{*}{ Item } & \multicolumn{2}{c|}{$\begin{array}{c}\text { Completamente/ } \\
\text { Parcialmente en } \\
\text { Desacuerdo }\end{array}$} & $\begin{array}{c}\text { Parcialmente } \\
\text { de Acuerdo }\end{array}$ & \multicolumn{2}{c|}{$\begin{array}{c}\text { Completamente } \\
\text { de Acuerdo }\end{array}$} \\
\cline { 3 - 9 } & & $\begin{array}{c}\text { Escuelas } \\
\text { MUN }\end{array}$ & $\begin{array}{c}\text { Escuelas } \\
\text { PS }\end{array}$ & $\begin{array}{c}\text { Escuelas } \\
\text { MUN }\end{array}$ & $\begin{array}{c}\text { Escuelas } \\
\text { PS }\end{array}$ & $\begin{array}{c}\text { Escuelas } \\
\text { MUN }\end{array}$ & $\begin{array}{c}\text { Escuelas } \\
\text { PS }\end{array}$ \\
\hline $\begin{array}{l}\text { Bajo/ } \\
\text { Medio } \\
\text { Bajo }\end{array}$ & Los profesores se sienten... & 7,5 & 6,9 & 13,8 & 31,2 & 78,7 & 61,9 \\
\cline { 2 - 9 } & Los profesores y directivos... & 8,6 & 10,3 & 20,7 & 10,1 & 70,6 & 79,7 \\
\hline Medio & Los profesores se sienten... & 18,9 & 21,1 & 12,2 & 9,8 & 69,0 & 69,1 \\
\cline { 2 - 9 } & Los profesores y directivos... & 12,3 & 14,6 & 17,8 & 19,9 & 69,9 & 65,5 \\
\hline
\end{tabular}

Estos resultados indican que respecto al mito de la diversidad de proyectos educativos sólo puede afirmarse que las escuelas PS entregan una oferta segmentada de acuerdo al estatus socioeconómico, donde los sectores menos privilegiados reciben una educación más autoritaria, con menor involucramiento del profesorado y menos orientada hacia la excelencia académica.

\section{AFIRMACION 2:}

\section{Las escuelas PS obtienen mejores resultados que las MUN, dado el mismo NSE}

Utilizando la información oficial correspondiente al SIMCE 2004 para estudiantes de octavo básico se calculó el puntaje de desempeño de las distintas escuelas según la metodología definida en el estudio FONIDE ya referido ${ }^{6}$. Al analizar estos datos se observa claramente que no existe una diferencia de rendimiento entre los colegios de dependencia MUN y PS más allá de la explicada por la diferencia de NSE. Si se comparan los desempeños de las escuelas dentro de cada segmento socioeconómico veremos que los resultados de las escuelas MUN son significativamente superiores a los de sus pares PS en el NSE Bajo, y son superiores aunque no existe una diferencia significativa en el NSE

se sienten en libertad de plantear sus desacuerdos conmigo; 5. Los profesores y directivos se sienten parte importante del mismo proyecto.

6 Para evaluar esta hipótesis se utilizaron los puntajes de desempeño (de acuerdo a la metodología del estudio FONIDE) del total de las escuelas con octavo básico a nivel nacional. Este puntaje se basa en los resultados del SIMCE 2004 para octavo básico. Se puede evaluar la hipótesis de que las escuelas PS obtienen mejores resultados más allá del efecto del NSE de los estudiantes de dos formas: La primera es comparar los resultados al interior de cada NSE, la segunda es utilizar un modelo de regresión en el que ambas variables se introduzcan simultáneamente. Ambos métodos han sido utilizados para entregar una respuesta completa. 
Medio-Bajo. En cambio, las escuelas PS tienen un desempeño significativamente superior en el segmento Medio. En los sectores medios es posible observar una diferencia que favorece a los PS, pero en el caso de los establecimientos medios-altos la ventaja también la tienen los colegios municipalizados. Es decir, dado un NSE similar, en términos de desempeño la gestión PS no representa una ventaja frente a la municipalizada.

Ante lo anterior podemos descartar completamente una tendencia general de la educación PS a obtener mejores resultados que la MUN. Aunque es imposible comprobarla con los datos utilizados aquí, la hipótesis más plausible es afirmar que a medida que aumenta el NSE de los estudiantes el diferencial de inversión entre escuelas MUN y PS aumenta también, y con ello los resultados de las escuelas PS. Sin embargo, en la medida en que nos acercamos a la competencia en igualdad de condiciones de inversión -como ocurre en el NSE bajo- la educación MUN obtiene una ventaja significativa. Dicho de otro modo, los resultados parecen indicar que si la misma inversión se canalizara a través de escuelas MUN los resultados serían superiores.

\section{AFIRMACION 3:}

Las escuelas PS realizan una mejor gestión escolar que las MUN, dado el mismo NSE

Esta afirmación fue contrastada a partir de las siguientes dimensiones de la gestión escolar que pudieron ser analizadas a partir del estudio FONIDE:

\section{a) Evaluaciones docentes ${ }^{7}$}

Respecto del uso sistemático de evaluaciones de los docentes, no se observan diferencias significativas entre escuelas PS y MUN. Como se observa en el cuadro siguiente los datos nos muestran pequeñas diferencias que de ser consideradas favorecerían a las escuelas municipalizadas.

\begin{tabular}{|l|c|c|c|}
\hline \multicolumn{4}{|c|}{$\begin{array}{c}\text { Indicadores sobre Evaluación Docente según Dependencia. } \\
\text { Medias y Frecuencia de Respuesta Afirmativa (\%) }\end{array}$} \\
\hline \multicolumn{1}{|c|}{ Indicador } & $\begin{array}{c}\text { Escuelas } \\
\text { MUN }\end{array}$ & $\begin{array}{c}\text { Escuelas } \\
\text { PS }\end{array}$ & Total \\
\hline $\begin{array}{l}\text { Número de Actores que participan en la } \\
\text { Evaluación }\end{array}$ & 3.187 & 3.145 & 3.174 \\
\hline $\begin{array}{l}\text { Número de Actores que participan en la } \\
\text { Evaluación excluyendo Profesores }\end{array}$ & 2.745 & 2.594 & 2.694 \\
\hline $\begin{array}{l}\text { Existe o no Evaluación de la Actividad Docente } \\
\% \text { afirmativo })\end{array}$ & 96,71 & 93,73 & 96,17 \\
\hline
\end{tabular}

7 Respecto del uso de evaluaciones de la actividad docente, el estudio utilizado permitía construir tres indicadores alternativos: a) el número de actores diferentes que participan en la evaluación de los profesores; b) el mismo indicador, con exclusión de los mismos docentes (autoevaluación), y c) la existencia o no de estas evaluaciones. Estos tres indicadores se utilizaron para evaluar la hipótesis de que en las escuelas PS se utilizan más estos mecanismos de gestión pedagógica. 


\section{b) Evaluaciones técnicas ${ }^{8}$}

Como se observa en el cuadro no hay diferencias estadísticamente significativas en este elemento de la gestión escolar en relación a dependencia MUN y PS. Los datos nos muestran pequeñas diferencias que de ser consideradas favorecerían a las escuelas municipalizadas.

\begin{tabular}{|l|c|c|c|}
\hline \multicolumn{4}{|c|}{ Número de evaluaciones técnicas por escuela en el último año. } \\
Según dependencia \\
\hline & Escuelas MUN & Escuelas PS & Total \\
\hline Media & 4.379 & 3.970 & 4.155 \\
\hline $\mathrm{N}$ & 123 & 112 & 235 \\
\hline
\end{tabular}

\section{c) La capacidad de gestionar recursos monetarios adicionales ${ }^{9}$}

Respecto de esta variable es posible observar que las escuelas municipalizadas obtienen resultados muy superiores a las escuelas PS, siendo ésta una diferencia significativa $(\mathrm{a}=0,0)$. Podemos decir que las escuelas municipalizadas poseen mayores capacidades para gestionar recursos externos adicionales que las escuelas PS, aun sin controlar por NSE, es decir, aun cuando en términos generales atienden a estudiantes con menores recursos.

\begin{tabular}{|c|c|c|}
\hline \multicolumn{3}{|c|}{$\begin{array}{c}\text { Porcentaje (\%) de escuelas que lograron gestionar recursos adicionales } \\
\text { a la Subvención según Dependencia }\end{array}$} \\
\hline Escuelas MUN & Escuelas PS & Total \\
\hline 55,67 & 23,58 & 38,06 \\
\hline
\end{tabular}

\section{d) Recursos para el fomento de la educación}

Respecto a la existencia y uso de recursos relacionados con el fomento de la educación, es evidente que hay una clara diferencia entre los establecimientos de acuerdo al nivel socioeconómico al que pertenecen. En el estudio se muestra que los establecimientos PS tienen más recursos en los ítemes de biblioteca, conexión a Internet, laboratorio de ciencias, sala de música, laboratorio de inglés y sala multimedia.

8 Respecto del uso de evaluaciones técnicas de la escuela, se utilizó como indicador el número de evaluaciones realizadas en el último año, con un tope superior de 10 que tiene el fin de limitar el efecto de los casos aberrantes.

9 Se utilizó como indicador sencillamente una variable binaria que indica si estos resultados adicionales fueron gestionados y conseguidos o no. El uso de indicadores más específicos, que incluyeran por ejemplo el monto de estos recursos, es cuestionable debido a la necesidad de considerar múltiples factores como el tamaño de la escuela, los recursos de la comunidad circundante, etc. 


\begin{tabular}{|l|c|c|c|c|c|}
\hline \multicolumn{7}{|c|}{$\begin{array}{c}\text { Porcentaje de escuelas (\%) que cuentan con diferentes Recursos Pedagógicos. } \\
\text { Según Dependencia o Nivel Socioeconómico }\end{array}$} \\
\hline \multirow{2}{*}{ Recurso } & Total & $\begin{array}{c}\text { Escuelas } \\
\text { MUN }\end{array}$ & $\begin{array}{c}\text { Escuelas } \\
\text { PS }\end{array}$ & $\begin{array}{c}\text { Escuelas } \\
\text { NSE } \\
\text { Bajo y } \\
\text { Medio-Bajo }\end{array}$ & $\begin{array}{c}\text { Escuelas } \\
\text { NSE } \\
\text { Medio }\end{array}$ \\
\hline Biblioteca & 87,66 & 86,66 & 89,98 & 84,73 & 90,40 \\
\hline Uso de computadores & 97,45 & 98,57 & 98,08 & 95,34 & 100,00 \\
\hline Conexión a Internet & 92,34 & 91,80 & 95,51 & 90,91 & 94,02 \\
\hline Laboratorios de ciencia & 46,81 & 37,77 & 60,29 & 33,89 & 53,11 \\
\hline Sala de música, instrumentos, etc. & 40,43 & 31,82 & 55,19 & 28,80 & 45,55 \\
\hline Laboratorio de Inglés & 11,49 & 5,26 & 21,93 & 5,86 & 16,65 \\
\hline Sala multimedia & 51,49 & 40,69 & 64,93 & 41,61 & 61,76 \\
\hline n (casos) & 235 & 123 & 112 & 106 & 105 \\
\hline
\end{tabular}

Al controlar según NSE, aparece que en los sectores medios-bajos y bajos el acceso a Bibliotecas, Computadores e Internet desde los establecimientos MUN es mayor que en los PS. Respecto de estos recursos las escuelas PS son una ventaja para los sectores medios, mientras que las MUN son ventajosas para los sectores Bajo y Medio-Bajo.

En el caso de los Laboratorios de Ciencia y de la sala multimedia aún en los sectores medios y bajos es mayor el acceso desde los establecimientos PS. Los establecimientos municipales declaran tener menos de estos recursos, en general y de manera transversal. Respecto de estos recursos entonces las escuelas PS se encuentran en ventaja siempre.

\begin{tabular}{|l|c|c|c|c|}
\hline \multicolumn{3}{|c}{$\begin{array}{c}\text { Porcentaje de escuelas (\%) que cuentan con diferentes Recursos Pedagógicos. } \\
\text { Según Dependencia y Nivel Socioeconómico }\end{array}$} \\
\hline \multirow{2}{*}{ Recurso } & \multicolumn{2}{c|}{$\begin{array}{c}\text { Escuelas NSE Bajo y } \\
\text { Medio-Bajo }\end{array}$} & \multicolumn{2}{c|}{ Escuelas NSE Medio } \\
\cline { 2 - 5 } & $\begin{array}{c}\text { Escuelas } \\
\text { MUN }\end{array}$ & $\begin{array}{c}\text { Escuelas } \\
\text { PS }\end{array}$ & $\begin{array}{c}\text { Escuelas } \\
\text { MUN }\end{array}$ & $\begin{array}{c}\text { Escuelas } \\
\text { PS }\end{array}$ \\
\hline Biblioteca & 84,28 & 86,37 & 88,96 & 90,98 \\
\hline Uso de computadores & 97,74 & 86,58 & 100,00 & 100,00 \\
\hline Conexión a Internet & 94,05 & 79,45 & 85,51 & 97,48 \\
\hline Laboratorios de ciencia* & 36,52 & 24,33 & 31,30 & 61,98 \\
\hline Sala de música, instrumentos, etc.* & 28,25 & 30,83 & 25,65 & 53,65 \\
\hline Laboratorio de Inglés** & 5,57 & 6,92 & 5,65 & 21,13 \\
\hline Sala multimedia** & 38,93 & 51,36 & 42,47 & 69,61 \\
\hline n (casos) & 79 & 27 & 38 & 67 \\
\hline
\end{tabular}

* Diferencia significativa entre dependencias.

** Diferencia significativa entre dependencias aún controlando por NSE. 


\section{e) Instancias de perfeccionamiento para profesores}

Para evaluar este elemento ocupamos dos indicadores presentes en el estudio: a) el uso de oportunidades de perfeccionamiento como forma de estímulo a los profesores, y b) la disponibilidad de asistencia técnica para los profesores.

La tabla siguiente muestra el porcentaje de escuelas en que se usa el perfeccionamiento como incentivo y el porcentaje de escuelas donde los profesores acceden constantemente a asistencia técnica. En ninguno de los casos hay diferencias significativas por dependencia. No hay diferencia entre escuelas PS y MUN en cuanto a las instancias de perfeccionamiento a los profesores.

\begin{tabular}{|l|c|c|c|}
\hline \multicolumn{4}{|c|}{$\begin{array}{c}\text { Escuelas con Acceso de los Profesores a Perfeccionamiento. } \\
\text { Frecuencias Relativas de Acceso (\%) }\end{array}$} \\
\hline \multicolumn{1}{|c|}{ Indicador } & Total & $\begin{array}{c}\text { Escuelas } \\
\text { MUN }\end{array}$ & $\begin{array}{c}\text { Escuelas } \\
\text { PS }\end{array}$ \\
\hline $\begin{array}{l}\text { Escuelas que usan Perfeccionamiento } \\
\text { como Incentivo }\end{array}$ & 70,64 & 71,02 & 74,01 \\
\hline $\begin{array}{l}\text { Escuelas donde los Profesores acceden } \\
\text { constantemente a Asistencia Técnica }\end{array}$ & 47,21 & 49,09 & 41,98 \\
\hline $\mathrm{n}$ (casos) & 235 & 123 & 112 \\
\hline
\end{tabular}

\section{f) Un fuerte liderazgo académico del director del establecimiento}

La encuesta utilizada recoge tres evaluaciones de la fuerza del liderazgo del directivo de cada escuela: la realizada por él/ella mismo/a, la realizada por el jefe de UTP y la realizada por un profesor jefe de $8^{\circ}$ básico. Se utiliza aquí como indicador de fuerza del liderazgo el porcentaje de escuelas en que los tres actores evalúan el liderazgo del directivo como "fuerte" o "muy fuerte".

Como se observa en el cuadro no hay diferencias estadísticamente significativas en este elemento de la gestión escolar en relación a dependencia MUN o PS. Los datos nos muestran pequeñas diferencias que de ser consideradas favorecerían a las escuelas municipalizadas.

\begin{tabular}{|l|c|c|c|}
\hline $\begin{array}{c}\text { Escuelas con Liderazgo Fuerte } \\
\text { (los tres entrevistados coinciden en que el directivo } \\
\text { posee un Liderazgo "Fuerte" o "Muy Fuerte") }\end{array}$ & Total & $\begin{array}{c}\text { Escuelas } \\
\text { MUN }\end{array}$ & $\begin{array}{c}\text { Escuelas } \\
\text { PS }\end{array}$ \\
\hline Porcentaje de Escuelas (\%) & 63,13 & 67,38 & 59,64 \\
\hline $\mathrm{n}$ (casos) & 235 & 123 & 112 \\
\hline
\end{tabular}

\section{g) Los mecanismos de control e incentivos}

Se han utilizado seis indicadores distintos para evaluar este aspecto: 1) el uso en la escuela de incentivos monetarios; 2) el monitoreo constante del desempeño de los 
estudiantes de los profesores; 3) el uso de supervisión en el aula; 4) la definición periódica de metas para los profesores; 5) la práctica de evaluar el problema del desempeño en todas las reuniones de profesores y directivos, y 6) el monitoreo del cumplimiento por los profesores de las planificaciones establecidas.

De todos estos indicadores, sólo existen diferencias significativas en uno: el uso de incentivos monetarios. Las escuelas PS utilizan significativamente más estímulos monetarios para sus profesores, lo que probablemente se asocie a restricciones institucionales en las escuelas municipales.

\begin{tabular}{|l|c|c|c|c|c|}
\hline \multicolumn{7}{|c|}{$\begin{array}{c}\text { Porcentaje (\%) de Escuelas que Utilizan distintos Mecanismos de Control e Incentivos. } \\
\text { Según Dependencia o Nivel Socioeconómico }\end{array}$} \\
\hline \multirow{2}{*}{ Mecanismo } & Total & $\begin{array}{c}\text { Escuelas } \\
\text { MUN }\end{array}$ & $\begin{array}{c}\text { Escuelas } \\
\text { PS }\end{array}$ & $\begin{array}{c}\text { Escuelas } \\
\text { NSE } \\
\text { Bajo y } \\
\text { Medio- } \\
\text { Bajo }\end{array}$ & $\begin{array}{c}\text { Escuelas } \\
\text { NSE } \\
\text { Medio }\end{array}$ \\
\hline Uso de Incentivos Monetarios* & 31,45 & 24,05 & 37,53 & 21,80 & 31,81 \\
\hline Monitorear desempeño estudiantes & 92,54 & 94,33 & 91,08 & 91,53 & 92,51 \\
\hline Supervisión en el aula & 81,54 & 82,08 & 81,10 & 85,14 & 84,36 \\
\hline Definición periódica de metas & 97,46 & 97,98 & 97,03 & 97,61 & 99,36 \\
\hline Revisión desempeño en reuniones & 40,72 & 37,59 & 43,30 & 41,24 & 40,95 \\
\hline Monitoreo cumplimiento planificación & 96,19 & 95,65 & 96,62 & 95,15 & 97,38 \\
\hline n (casos) & 235 & 123 & 112 & 106 & 105 \\
\hline
\end{tabular}

* Diferencia significativa.

Sin embargo, si comparamos el porcentaje de escuelas que utilizan estos incentivos monetarios dentro de cada NSE encontramos que sólo en el sector Medio esta diferencia sigue siendo significativa. Es decir, los estudiantes de NSE Bajo y Medio-Bajo no se benefician realmente de esta ventaja de las escuelas PS y para ellos no hay diferencia alguna en términos de incentivos o mecanismos de control según el tipo de escuela al que asistan.

\begin{tabular}{|c|c|c|c|}
\hline \multicolumn{3}{|c|}{$\begin{array}{c}\text { Porcentaje (\%) de Escuelas que Utilizan Incentivos Monetarios } \\
\text { para los Profesores. }\end{array}$} \\
Según Dependencia y Nivel Socioeconómico \\
\hline \multicolumn{2}{|c|}{$\begin{array}{c}\text { Escuelas NSE } \\
\text { Bajo y Medio-Bajo }\end{array}$} & \multicolumn{2}{c|}{ Medio } \\
\hline $\begin{array}{c}\text { Escuelas } \\
\text { MUN }\end{array}$ & $\begin{array}{c}\text { Escuelas } \\
\text { PS }\end{array}$ & $\begin{array}{c}\text { Escuelas } \\
\text { MUN }\end{array}$ & $\begin{array}{c}\text { Escuelas } \\
\text { PS }\end{array}$ \\
\hline 21,92 & 21,37 & 26,62 & 33,91 \\
\hline
\end{tabular}




\section{CONCLUSIONES}

La comparación en torno a datos estadísticos realizada en este estudio para verificar la existencia de diferencias entre establecimientos municipales y particulares subvencionados, dejó establecido que:

a) Los establecimientos particulares subvencionados diferencian sus proyectos escolares focalizándose según estrato socioeconómico. A partir de ello los sectores menos privilegiados reciben una educación más autoritaria, con menor involucramiento del profesorado y menos orientada hacia la excelencia académica, mientras los sectores medios y medios-altos reciben una educación orientada hacia la excelencia académica. Los establecimientos municipales son efectivamente más homogéneos pues sus proyectos apuntan transversalmente a la formación ciudadana y la excelencia académica, sin importar el estrato social al cual estén educando.

b) Controlando la variable NSE en términos de desempeño escolar la gestión PS no representa una ventaja frente a la municipalizada. Podríamos inferir entonces que si la misma inversión se canalizara a través de escuelas MUN los resultados serían superiores.

c) En gestión escolar no existirían diferencias significativas entre escuelas PS y MUN en: la evaluación sistemática de la actividad docente, el uso de evaluaciones técnico-pedagógicas de los establecimientos, instancias de perfeccionamiento para profesores, liderazgo académico del director del establecimiento, mecanismos de control e incentivos. En cuanto a la obtención de recursos monetarios adicionales a las subvenciones a través de gestiones especiales, los establecimientos municipalizados se muestran más eficientes y respecto a la existencia de recursos para el fomento de la educación están claramente relacionados con el NSE del establecimiento, donde en los sectores bajos y medios bajos existiría un mayor acceso a bibliotecas, computadores e Internet a través de los colegios municipales, mientras que en los particulares subvencionados habría mayor acceso a laboratorio de ciencias y/o sala multimedia.

Es decir, si incorporamos la variable socioeconómica en el análisis del panorama educativo en Chile, podemos afirmar que la gestión privada de la educación pública sólo ha reproducido la segmentación social del país, mostrándose poco efectiva a la hora de obtener resultados a medida que debe enfrentar contextos de mayores dificultades sociales y económicas. Ante lo anterior se favorece la hipótesis de que las escuelas de administración pública son instituciones de mayor flexibilidad, aprendizaje y capacidad para asumir contextos caracterizados por una serie de vulnerabilidades sociales de base, cuestión que abre una serie de desafíos en relación a la formulación de la política nacional de educación que parece haber invisibilizado seriamente las diferencias estructurales al comparar establecimientos municipales y particulares subvencionados sólo a partir del dato SIMCE, sin observar los mecanismos de selección claramente diferenciados que sí puede aplicar la educación de administración privada. 


\section{REFERENCIAS BIBLIOGRAFICAS}

Aedo, C. y Sapelli, C. (2001). "El sistema de vouchers en educación. Una revisión de la teoría y evidencia empírica para Chile", Estudios Públicos 82, otoño.

Brunner, J.J. y Elacqua, G. (2003). "Factores que inciden en una educación efectiva: Evidencia internacional”, en Hevia, R. (ed.) (2003). La Educación en Chile hoy, Ediciones Universidad Diego Portales, Santiago.

Carnoy, M. (2005). "La Búsqueda de la igualdad a través de las políticas educativas: alcances y límites", en García Huidobro (ed.). Políticas Educativas y Equidad, Reflexiones del Seminario Internacional sobre Políticas Educativas y Equidad, UNESCO-FORD-UNICEF, Santiago.

Cornejo, R. (2006). "El experimento educativo Chileno 20 años después: una mirada crítica a los logros y falencias del sistema escolar". REICE - Revista Electrónica Iberoamericana sobre Calidad, Eficacia y Cambio en Educación, 2006, Vol. 4, № 1, pp. 118-129.

Descouvieres, C., Redondo, J.M., Rojas, K. (2004). Un Estudio de la Eficiencia de la Educación Básica en Chile y su Posible Aplicación a la Evaluación de la Carrera Docente. Proyecto Did.S05-97/2. En Equidad y Calidad de la Educación en Chile. http://www.Opech.Cl/Inv/ Investigaciones/Equidadycalidad.Pdf

Elacqua, G. (2004). "El consumidor de la educación: El actor olvidado de la libre elección de colegios en Chile", Universidad Adolfo Ibáñez, Santiago.

Gallego, F.A. (2002). "Competencia y Resultados Educativos: Teoría y Evidencia para Chile," Working Papers Central Bank of Chile 150, Central Bank of Chile.

García-Huidobro, J.E. y Bellei, C. (2006). “¿Remedio para la inequidad? La Subvención escolar preferencial, en Revista Mensaje, marzo-abril, Santiago.

Gobierno de Chile. Proyecto de ley que establece una subvención escolar preferencial para estudiantes y niñas socioeconómicamente vulnerables, octubre 18 de 2005, mensaje $\mathrm{N}^{\circ}$ 362-353.

González, P., Mizala, A. y Romaguera, P. (2001). "Recursos diferenciados para la educación subvencionada en Chile", mimeo. Centro de Economía Aplicada. Universidad de Chile. Santiago.

Larrañaga, O. (2004). Competencia y participación privada: la experiencia chilena en educación. Estudios Públicos, 96, 107-144.

López, N. (2005). "Equidad educativa y desigualdad social. Desafíos de la educación en el nuevo escenario latinoamericano", IIPE, Buenos Aires.

Mizala, A. \& Romaguera, P. (2002). "Equity and Educational Performance". Documentos de Trabajo $\mathrm{N}^{\circ}$ 136, CEA, U. de Chile.

Mizala, A. y Romaguera, P. (2002). "Evaluación del desempeño e incentivos en la educación chilena", Cuadernos de Economía, Año 39, N 118, diciembre de 2002.

Mizala, A. y Romaguera, P. (2001). "Regulación, incentivos y remuneraciones de los profesores en Chile". Documentos de Trabajo N ${ }^{\circ} 116$, CEA, U. de Chile.

Mizala, A. \& Romaguera, P. (2000). "School performance and choice: the Chilean experience", Journal of Human Resources, vol. XXXV, number 2, spring 2000.

Mizala, A. y Romaguera, P. (2000). "Sistemas de incentivos en educación y la experiencia del Sned en Chile". Documentos de Trabajo No 82, CEA, U. de Chile.

OPECH (2006). "Sistema de medición de la calidad de la educación SIMCE: Balance crítico y proyecciones imprescindibles". Documento de Trabajo $N^{\circ} 1$. Santiago de Chile.

Redondo, J.M. (2000). "El fracaso escolar y las funciones estructurales de la escuela: una perspectiva crítica". Revista de Sociología № 14. FACSO. Universidad de Chile, pp. 7-23.

Redondo, J.M. y Descouvieres, C. (2000). "Eficacia y Eficiencia en las escuelas básicas chilenas (1990-1997). Un estudio de valor agregado". I Jornadas de Psicología Educacional (2000), La Serena: Universidad de La Serena, pp. 14-24.

Redondo, J.M. y Descouvieres, C. (2001). "Eficacia y Eficiencia de las escuelas básicas chilenas (1990-1997). Aproximaciones a un estudio de valor agregado". Revista Enfoques Educacionales 
Vol. N N $^{\circ}$. N 1 (2000-2001). Departamento de Educación. FACSO. Universidad de Chile, pp. 139-154. 2001.

Redondo, J. M. (2005). "El experimento Chileno en educación: ¿Conduce a mayor equidad y calidad en la educación? Ultima década $\mathrm{N}^{\circ}$ 22, CIDPA Valparaíso, agosto 2005, pp. 95-110.

Revista Persona y Sociedad (2003). Cambio Educacional: actores y políticas. Vol. XVII, No 1 , abril 2003. Ilades y Universidad Alberto Hurtado. Santiago de Chile.

Sapelli, C. y Torche, A. (2002). Subsidios al alumno o a la escuela: efectos sobre la elección de colegios públicos. Cuadernos de Economía, 39 (117), 175-202.

Sapelli, C. \& Vial, B. (2002). The performance of private and public schools in the Chilean voucher system. Cuadernos de Economía, 39 (118), 423-454. Enlace creado en enero 2007.

Sapelli, C. \& Vial, B. (2005). Peer effects and relative performance of voucher schools in Chile. Documento de Trabajo No 256, Instituto de Economía, P. Universidad Católica de Chile. Descargado en enero 2007.

Sapelli, C. \& Vial, B. (2005). Private vs. public voucher schools in Chile: New evidence on efficiency and peer effects. Documento de Trabajo No 289, Instituto de Economía, P. Universidad Católica de Chile. Descargado en enero 2007.

Tokman, A. (2002). Is private education better? Evidence from Chile. Banco Central, Documento de Trabajo $N^{\circ} 147$. Descargado en enero 2007. 